\title{
CHECK-LIST DA AVIFAUNA E INVENTÁRIO DA FLORA ARBÓREA DAS ÁREAS DE USO COMUM DA ETEC PROF. DR. ANTONIO EUFRÁSIO DE TOLEDO, PRESIDENTE PRUDENTE-
} SP

\section{Marcelo Wendeborn Miranda de Oliveira ${ }^{1}$}

Silvério Takao Hosomi ${ }^{2}$

RESUMO: As aves são, em geral, os vertebrados terrestres mais conspícuos nas paisagens naturais ou artificiais que nos cercam. É possível observar uma boa variedade de espécies até mesmo dentro das cidades mais populosas. O inventário das plantas arbóreas e o levantamento das espécies de aves que frequentam o ambiente da ETEC Prof. Dr. Antonio Eufrásio de Toledo tem como finalidade embasar atividades de Educação Ambiental dentro do espaço escolar, além de contribuir para a valorização do seu espaço verde. O levantamento da avifauna foi realizado nos meses de abril a maio de 2012 por análise qualitativa através de observações visuais, sonoras e também através de registros em câmara fotográfica. As espécies arbóreas com DAP (Diâmetro a altura do peito) maior que $10,0 \mathrm{~cm}$ e localizadas nos espaços de uso comum (Horta, Viveiro de mudas, Alojamentos, Administração e Salas de aulas) da ETEC foram inventariadas, sendo coletadas informações como altura da árvore, altura do fuste, altura da copa e DAP. No levantamento de avifauna totalizou-se um esforço amostral de 28 horas, durante as quais foram observadas 48 espécies de aves distribuídas em 25 famílias, sendo as mais frequentes Columbidae (24\%), Emberizidae (20\%) e Psittacidae (16\%). No inventário

\footnotetext{
${ }^{1}$ Biólogo, Alta Sorocabana/APTA, Bolsista de treinamento técnico. E-mail.marcelo_wendeborn@hotmail.com ${ }^{2}$ Biólogo, ETEC Prof. Dr. Antonio Eufrásio de Toledo, Docente. E-mail. silveriohosomi@gmail.com
} 
de plantas arbóreas foram registrados 367 indivíduos, sendo as famílias Fabaceae (23,4\%), Myrtaceae (19.9\%) e Bignoniaceae (6,8\%) as mais freqüentes. Entre as espécies encontradas, 52,7\% correspondem a exóticas. Esses dados revelam a importância da conservação e enriquecimento da área verde para a manutenção da avifauna que adotou a ETEC como refúgio.

Palavras chave: Biodiversidade. Dispersão. Aves.

\section{INTRODUÇÃO}

De acordo com Fontana et al (2003), as aves são os vertebrados terrestres mais notáveis nas paisagens artificiais ou naturais que nos rodeiam, sendo possível observar grande variedade de espécies até mesmo dentro das cidades mais populosas. Devido a essa convivência próxima e quase sempre equilibrada e harmoniosa com o ser humano, as aves talvez sejam as criaturas mais admiradas, observadas, e protegidas de todas as formas de vida terrestre existentes.

Segundo Matos (2011), a grande facilidade de identificar espécies de aves torna viável sua utilização como bioindicadores da qualidade ambiental e do grau de perturbação dos ecossistemas naturais, especialmente devido ao uso de métodos pouco invasivos como a fotografia in loco e a observação através de binóculo, com possível identificação em campo por pessoas de qualquer nível de escolaridade.

A classe das Aves é a mais notada e melodiosa sendo, para alguns, a mais bonita dentre os vertebrados conhecidos (HICKMAN, ROBERTS, LARSON, 2004). Esses animais são considerados por muitos o símbolo de paz e amor pela humanidade (GRILL 1995).

Ainda segundo Vuilleumier (2008) as aves possuem características especiais que as diferenciam de outros seres, como penas, membros em forma de asas, ossos pneumáticos que o possibilitam seu vôo, além serem endotérmicas e estarem distribuídas em grande parte do globo terrestre.

São reconhecidas pela comunidade científica cerca de 9.800 espécies de aves no mundo (CLEMENTS, 2007). A América do Sul, por possuir diversos biomas com características bem distintas, é o local onde há maior diversidade da classe em questão 
(RIDGELY \& TUDOR, 1994). Com cerca de 1.700 espécies o Brasil possui a terceira avifauna mais rica do mundo (MARÇAL JÚNIOR; FRANCHIN, 2003). O Comitê Brasileiro de Registros Ornitológicos apresenta o Brasil atualmente, com aproximadamente 1832 espécies de aves catalogadas (CBRO, 2011).

Por possuir grande diversidade ambiental, com relevos e vegetações diversificados, o estado de São Paulo é grande colaborador para tamanha riqueza de espécies de aves brasileiras, e sem dúvida é na Mata Atlântica que se encontra a maior quantidade de espécies (SILVA \& ALEIXO, 1996).

O bioma Cerrado é a maior, a mais rica e, provavelmente, a mais ameaçada savana tropical do mundo (SILVA \& BATES, 2002). É conhecida como a segunda maior ecorregião do Brasil, abrangendo $25 \%$ do território nacional. Apresenta alto endemismo em relação à flora, diversidade de vertebrados consideravelmente alta, e ocupa o quarto lugar no mundo em diversidade de aves (PROBIO, 2002).

Pesquisas mostram que em um ecossistema natural as aves servem como indicadoras de um ambiente funcional e saudável. Esses animais possuem um grande valor, pois contribuem em diversos aspectos, como por exemplo, no controle biológico de pragas, carrapatos, cobras, formigas, ratos entre outros. Também contribuem na polinização de flores e disseminação de sementes, nos ajudando a viver cada dia melhor (ANDRADE, 1993).

O município de Presidente Prudente, localizado ao oeste do Estado de São Paulo, já foi coberto pelos biomas do Cerrado e Mata Atlântica. Essa cobertura vegetal, no entanto, tem sido constantemente destruída pela ocupação humana. A partir do século XVIII iniciou-se na região uma busca desenfreada por minerais preciosos, o que levou à transformação de áreas até então florestadas em extensas regiões para 0 desenvolvimento da agricultura e pecuária (RIECHELMANN, 2006).

No final do século XIX, o café passou a ser o principal produto brasileiro para exportação e no fim do mesmo século o cultivo de cana-de-açúcar estimulou a busca de novas terras produtivas. A partir daí o território paulista perdeu grande parte da sua cobertura florestal por sofrer um intenso processo de desmatamento, tendo sido destruídos $89,5 \% \quad(15.776,848$ ha) das formações florestais em pouco tempo (RIECHELMANN, 2006). 
A grande interferência humana nos ambientes florestais para 0 desenvolvimento da agropecuária e estabelecimento de áreas urbanas e distritos industriais têm trazido diversas conseqüências as aves, pois não é adequado preservar uma rica avifauna em pequenas sobras de habitat, já que algumas espécies exigem ambientes amplos (SICK, 1997).

Entre as aves da família Columbidae, há trezentas e nove espécies que habitam desde florestas densas até desertos, das regiões temperadas aos trópicos (BAPTISTA et al., 1997). De acordo com Sick (1997), esse grupo está distribuído por todos os biomas brasileiros, podendo procriar o ano todo. A postura de ovos varia nas espécies entre um e três, o período de incubação entre 11 e 19 dias, sendo os filhotes nidícolas e alimentados pelos pais com o "leite de papo", massa queijosa composta pelo epitélio digestivo do papo, que é fortemente desenvolvido em ambos os sexos durante a época de criação.

Em levantamento avifauna realizado em uma área de Cerrado no Bairro do Central Parque na cidade de Sorocaba/SP, foram observadas 75 espécies de aves que segundo Sick (1997), representam 4,6\% das aves existentes no Brasil, abrangendo 30 famílias, sendo que as famílias com as maiores riquezas de espécies foram Tyrannidae, Columbidae e Picidae. Observou-se a presença de membros da família Columbidae, como o pombão (Patagioenas picazuro), a rolinha-roxa (Columbina talpacoti), pomba-debando (Zenaida auriculata), fogo-apago (Columbina squammata) e juriti-pupu (Leptotila verreauxI) (SILVA \& NAKANO, 2008).

Em estudos realizados sobre a distribuição espacial de aves em um fragmento florestal do campus da Universidade Estadual de Londrina/PR, foram registradas 288 espécies de aves distribuídas em diferentes famílias e ordens. Entre os membros da família Columbidae estavam presentes as espécies Columba picazuro, Columba cayennensis, Zenaida auriculata, Columbina picui, Columbina talpacoti, Leptotila verreauxi, Leptotila rulaxila (GIMENES \& ANJOS, 2000).

O levantamento de avifauna no campus da Universidade Estadual do Centro-Oeste de Guarapuava/PR observou um total de 125 espécies de aves, distribuídas em 42 famílias e 16 ordens. Entre os membros da família Columbidae estavam presentes as espécies Columbina talpacoti, Columbina picui, Columba livia, Patagioenas picazuro, Zenaida auriculata, Leptotila verreauxi, Leptotila rufaxilla (VOGEL et al. 2011) 
Os estudos também mostram como elas interagem com a atividade antrópica e como esta atividade se torna dependente dessas aves, já que esses animais contribuem para a o controle de pragas existentes em áreas urbanas e/ou com forte atividade humana (VOGEL et al. 2011)

O levantamento das espécies de aves que frequentam o ambiente da ETEC Prof. Dr. Antonio Eufrásio de Toledo teve como finalidade permitir o embasamento de atividades de Educação Ambiental dentro do espaço escolar, além de contribuir para a valorização do seu espaço verde.

\section{DESENVOLVIMENTO}

O presente trabalho foi realizado na área da Escola Técnica - ETEC Prof. Dr. Antonio Eufrásio de Toledo, pertencente ao Centro Estadual de Educação Tecnológica Paula Souza (CEETEPS), Presidente Prudente - SP, situado a $22^{\circ} 10^{\prime} 32$ "S de latitude e $51^{\circ} 22$ '37"O de longitude, as margens da Rodovia Raposo Tavares no Km 561, distante 10 Km do Centro de Presidente Prudente e com uma área total de 100 alqueires.

O levantamento da avifauna foi realizado nos meses de abril e maio de 2012 por análise qualitativa através de observações visuais e sonoras, com o auxílio de binóculo (Pentax $10 \times 25 \mathrm{~mm}$ ) e também através de registros fotográficos utilizando uma câmara fotográfica (Canon EOS 40D, lentes 28-75 mm e 75-300mm). Todos os horários de observações foram registrados em caderno de campo para cálculo do índice de abundância, sendo as observações realizadas por caminhadas e ponto fixo em áreas de uso comum da ETEC, totalizando um esforço amostral de 28 horas.

Para identificação das espécies fotografadas foram utilizadas referências especializadas. As espécies arbóreas localizadas nos espaços de uso comum da ETEC com DAP (Diâmetro a altura do peito) maior que 10,0 cm foram inventariadas, sendo coletadas informações como altura da árvore, altura do fuste, altura da copa e DAP.

As observações realizadas permitiram identificar 48 espécies de aves, o que segundo Andrade (1993) e Sick (1997) representa 2,76\% das aves existentes no Brasil. De acordo com o que se pode observar na Figura 1, os espécimes identificados distribuem-se em 15 ordens e 25 famílias, sendo as mais frequentes Columbidae (24\%), 
Emberizidae (20\%) e Psittacidae (16\%). As aves não Passeriformes foram as mais abundantes, representando $57 \%$ das espécies observadas (figura 2).

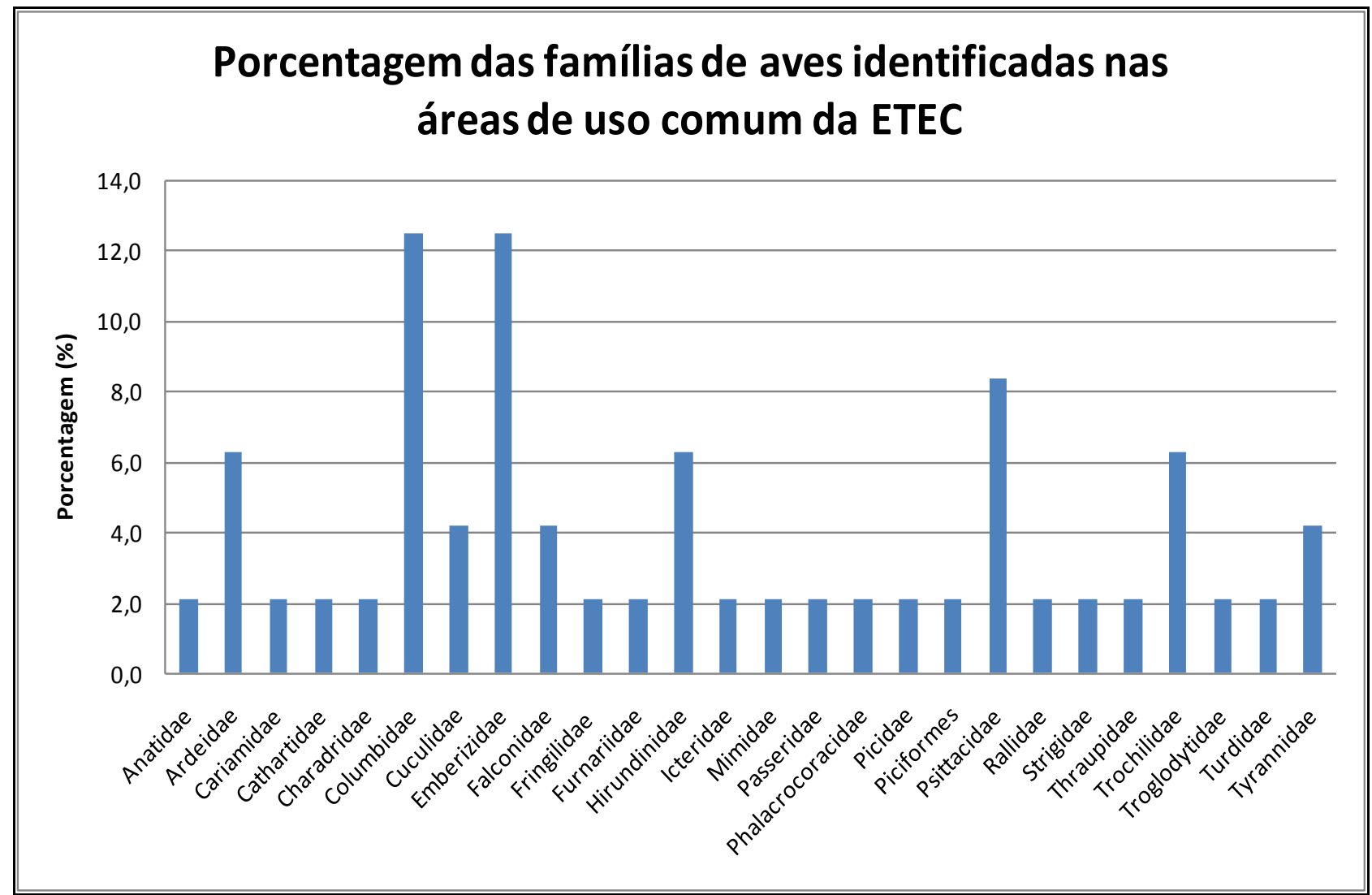

Figura 1: Freqüência relativa (\%) de famílias de aves encontradas nas áreas de uso comum da ETEC de Presidente Prudente. Fonte: Os autores

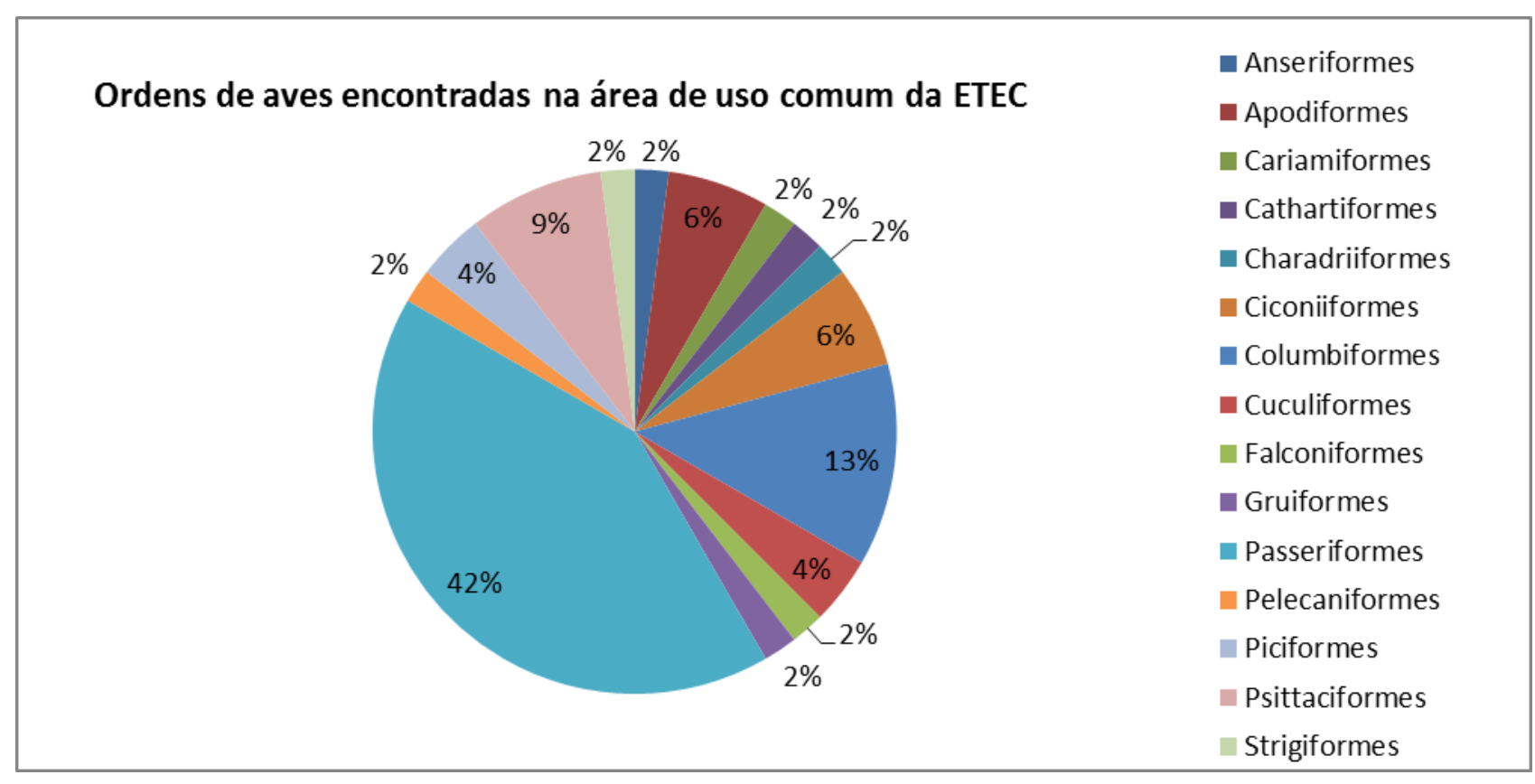


Figura 2: Freqüência relativa (\%) de ordens de aves de aves encontradas nas áreas de uso comum da ETEC. Fonte: Os autores

Como pode ser visto na Figura 3, com relação às guildas tróficas exploradas pelas aves houve predomínio de onívoras e insetívoras, com 15 espécies (31\%) e 12 espécies (17\%), respectivamente, sendo tais resultados um reflexo do ambiente sazonal. Segundo Willis (1976) e D'Angelo-Neto et al. (1998), a onivoria é uma categoria trófica comum e oportunista em áreas abertas e sob influência antrópica, uma vez que representa um efeito tampão contra flutuações no suprimento de alimentos.

\section{Guildas tróficas (\%) exploradas pelas aves na ETEC}

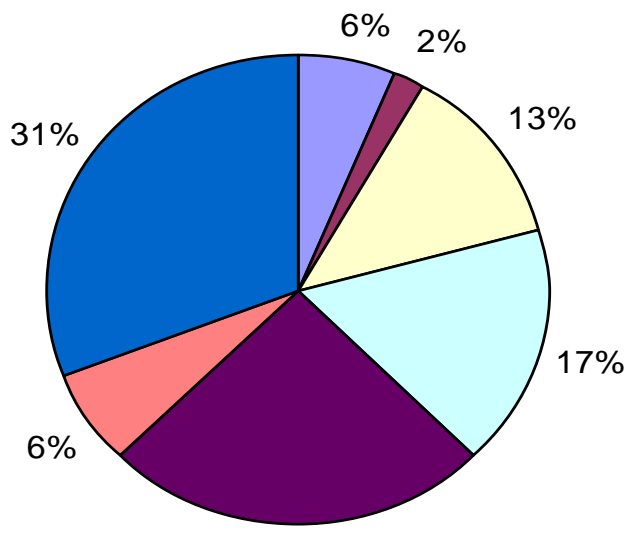

$\square$ Carnivora
$\square$ Detritívoro
$\square$ Frugívoro
$\square$ Granívoro
$\square$ Insetívoro
$\square$ Nectarívoro
$\square$ Onívoro

$25 \%$

Figura 3: Guildas tróficas (\%) exploradas pelas aves na ETEC de Presidente Prudente. Fonte: Os autores

Por fim, estabeleceu-se um check-list das aves da ETEC Prof. Dr. Antônio Eufrásio de Toledo (Tabela 1), o que irá permitir um acompanhamento da diversidade ao longo do tempo.

Tabela 1: Check-list das espécies de aves registradas no campus da ETEC Prof. Dr. Antonio Eufrásio de Toledo.

\begin{tabular}{|c|c|c|c|c|c|}
\hline № & Nome Popular & Nome Científico & Família & Ordem & Guilda \\
\hline 1 & Andorinha & Pygochelidon cyanoleuca & Hirundinidae & Passeriformes & Insetívoro \\
\hline 2 & Andorinha azul e branca & Notiochelidon cyanoleuca & Hirundinidae & Passeriformes & Insetívoro \\
\hline
\end{tabular}


ISSN 1980-0827

3 Andorinha de rabadilha branca

4 Anu branco

5 Anu preto

6 Beija flor fada

7 Beija flor tesoura roxeado

8 Beija flor tesoura

9 Bem-te-vi

10 Biguá

11 Canário da terra

12 Canário rasteiro

13 Caturrita

14 Chopim gaudério

15 Corruíra do campo

16 Coruja buraqueira

17 Frango d'água carijó

18 Garça branca grande

19 Garça vaqueira

20 Gaturamo verdadeiro

21 Gavião carcará

22 João de barro

23 Lavadeira mascarada

24 Maritaca

25 Marreca sará

26 Papa capim de coleira

27 Pardal

28 Periquito de asa amarela

29 Pica pau carijó

30 Pica pau cabeça amarela

31 Pomba amargosa

32 Pomba asa branca / Pombão

33 Pomba botafogo

34 Pomba de bando- Avoante

35 Quero quero

36 Rola Fogo apagou

37 Rolinha roxa

38 Sabiá do campo

39 Sabiá poca

40 Sabiá da mata

41 Sanhaçu cinza

42 Seriema

43 Socozinho

44 Tico tico do campo

45 Tico-tico

46 Tiziu
Tachycinea leucorrhoa

Guira guira

Crotophaga ani

Heliothrix aurita

Thalurania furcata

Eupetomena macroura

Pitangus sulphuratus

Phalacrocorax olivaceus

Sicalis flaveola

Sicalis citrina

Myiopsitta monachus

Molothrus bonariensis

Cistothorus platensis

Athene cunicularia

Porohyriops melanops

Ardea alba

Bubulcus ibis

Euphonia violaceae

Polyborus plancus

Furnarius rufus

Fluvicola nengeta

Brotogeris tirica

Ana discors

Dolospingus fringilloides

Passer domesticus

Brotogeris versicolurus

Colaptes melanochloros

Celeus flavescens

Columba plumbea

Columba picazuro

Patagioenas subvinacea

Zenaida auriculata

Vanellus chilensis

Columbina squammata

Columbina talpacoti

Mimus saturninus

Turdus amaurochalinus

Turdus fumigatus

Thraupis sayaca

Cariama cristata

Butorides striatus

Ammodramus humeralis

Zonotrichia capensis

Volatinia jacarina
Hirundinidae

Cuculidae

Cuculidae

Trochilidae

Trochilidae

Trochilidae

Tyrannidae

Phalacrocoracidae

Emberizidae

Emberizidae

Psittacidae

Icteridae

Troglodytidae

Strigidae

Rallidae

Ardeidae

Ardeidae

Fringilidae

Falconidae

Furnariidae

Tyrannidae

Psittacidae

Anatidae

Emberizidae

Passeridae

Psittacidae

Picidae

Picidae

Columbidae

Columbidae

Columbidae

Columbidae

Charadriidae

Columbidae

Columbidae

Mimidae

Turdidae

Turdidae

Thraupidae

Cariamidae

Ardeidae

Emberizidae

Emberizidae

Emberizidae
Passeriformes

Cuculiformes

Cuculiformes

Apodiformes

Apodiformes

Adopiformes

Passeriformes

Pelecaniformes

Passeriformes

Passeriformes

Psittaciformes

Passeriformes

Passeriformes

Strigiformes

Gruiformes

Ciconiiformes

Ciconiiformes

Passeriformes

Falconiformes

Passeriformes

Passeriformes

Psittaciformes

Anseriformes

Passeriformes

Passeriformes

Psittaciformes

Piciformes

Piciformes

Columbiformes

Columbiformes

Columbiformes

Columbiformes

Charadriiformes

Columbiformes

Columbiformes

Passeriformes

Passeriformes

Passeriformes

Passeriformes

Cariamiformes

Ciconiiformes

Passeriformes

Passeriformes

Passeriformes
Insetívoro

Insetívoro

Insetívoro

Nectarívoro

Nectarívoro

Nectarívoro

Onívoro

Carnívoro

Granívoro

Granívoro

Frugívoro

Onívoro

Insetívoro

Insetívoro

Onívoro

Carnívoro

Insetívoro

Onívoro

Onívoro

Insetívoro

Insetívoro

Frugívoro

Onívoro

Onívoro

Onívoro

Frugívoro

Insetívoro

Insetívoro

Frugívoro

Granívoro

Frugívoro

Granívoro

Onívoro

Granívoro

Granívoro

Onívoro

Onívoro

Onívoro

Onívoro

Onívoro

Carnívoro

Granívoro

Onívoro

Granívoro 


\begin{tabular}{|c|c|c|c|c|}
\hline 47 Tuim santo & Forpus passerinus & Psittacidae & Psittaciformes & Frugívoro \\
\hline
\end{tabular}
Fonte: Os autores

As Tabelas 2, 3 e 4 apresentam o check-list da flora arbórea elaborado na ETEC Prof. Dr. Antonio Eufrásio de Toledo. A localização e identificação das espécies existentes no local podem promover futuramente um manejo adequado dos indivíduos, de modo a favorecer uma maior ocupação pela avifauna.

Tabela 02: Dados das espécies arbóreas localizadas em cada área de uso comum da ETEC Prof. Dr. Antônio Eufrásio de Toledo.

\begin{tabular}{|c|c|c|c|c|c|c|c|c|}
\hline Nome popular & Nome científico & Família & Tipo & $\mathbf{N}$ & $\%$ & CAP & DAP & $\mathrm{H}$ tota \\
\hline \multicolumn{9}{|c|}{ Área dos Alojamentos $11.502 \mathrm{~m}^{2}$} \\
\hline Aroeira-pimenteira & Schinus terebinthifolius & Anacardiaceae & Nativa & 1 & 1,3 & 40,60 & 12,92 & 8,33 \\
\hline Carambola & Averrhoa carambola & Oxalidaceae & Exótica & 8 & 10,7 & 39,63 & 12,61 & 3,30 \\
\hline Cedro & Toona ciliata & Meliaceae & Exótica & 4 & 5,3 & 112,95 & 35,95 & 12,00 \\
\hline Coqueiro & Cocos nucifera & Arecaceae & Nativa & 1 & 1,3 & 62,00 & 19,74 & 5,68 \\
\hline Eucalipto & Eucalyptus sp. & Myrtaceae & Exótica & 2 & 2,7 & 86,65 & 27,58 & 17,23 \\
\hline Flamboyant & Delonix regia & Fabaceae & Exótica & 1 & 1,3 & 263,00 & 83,72 & 15,44 \\
\hline Goiaba & Psidium guajava & Myrtaceae & Nativa & 18 & 24,0 & 45,34 & 14,43 & 3,57 \\
\hline Ipê & Handroanthus sp. & Bignoniaceae & Nativa & 1 & 1,3 & 66,50 & 21,17 & 9,59 \\
\hline Jatobá & Hymenaea courbaril & Fabaceae & Nativa & 1 & 1,3 & 136,50 & 43,45 & 16,04 \\
\hline Mangueira & Mangifera indica & Anacardiaceae & Exótica & 4 & 5,3 & 68,64 & 21,85 & 7,97 \\
\hline Monguba & Pachira aquática & Bombacaceae & Nativa & 3 & 4,0 & 53,83 & 17,14 & 8,34 \\
\hline Oiti & Licania tomentosa & Chrysobalanaceae & Nativa & 12 & 16,0 & 61,75 & 19,65 & 9,10 \\
\hline Pata-de-Vaca & Bauhinia forticata & Fabaceae & Nativa & 13 & 17,3 & 75,58 & 24,06 & 7,66 \\
\hline Sibipiruna & Caesalpinia pluviosa & Fabaceae & Nativa & 1 & 1,3 & 129,60 & 41,24 & 18,13 \\
\hline Tipuana & Tipuana tipu & Fabaceae & Nativa & 5 & 6,7 & 235,17 & 74,86 & 18,27 \\
\hline Total & & & & 75 & 100,0 & - & - & - \\
\hline \multicolumn{9}{|c|}{ Área Caminho Bovinos $1.673,0 \mathrm{~m}^{2}$} \\
\hline Abacate & Persea Americana L. & Lauraceae & Exótica & 2 & 3,0 & 109,00 & 34,70 & 10,20 \\
\hline Angico & Albizia polycephala & Fabaceae & Nativa & 1 & 1,5 & 128,00 & 40,70 & 16,80 \\
\hline Cedro & Cedrela fissilis & Meliaceae & Exótica & 3 & 4,5 & 115,00 & 36,61 & 11,10 \\
\hline Farinha-seca & Albizia hasslerii & Fabaceae & Nativa & 1 & 1,5 & 340,00 & 108,20 & 17,70 \\
\hline Flamboyant & Delonix regia & Fabaceae & Exótica & 1 & 1,5 & 210,00 & 66,80 & 12,30 \\
\hline Goiaba & Psidium guajava & Myrtaceae & Nativa & 4 & 6,0 & 69,50 & 22,12 & 5,70 \\
\hline Jabuticaba & Myrciaria cauliflora & Myrtaceae & Nativa & 2 & 3,0 & 49,35 & 15,71 & 7,70 \\
\hline Jaca & Artocarpus integrifolia & Moraceae & Exótica & 5 & 7,5 & 114,46 & 36,43 & 11,22 \\
\hline Jatobá & Hymenaea courbaril & Fabaceae & Nativa & 2 & 3,0 & 97,80 & 31,13 & 14,80 \\
\hline Mangueira & Mangifera indica & Anacardiaceae & Exótica & 12 & 17,9 & 112,00 & 35,65 & 11,70 \\
\hline Pata-de-vaca & Bauhinia forticata & Fabaceae & Nativa & 1 & 1,5 & 65,00 & 20,70 & 5,30 \\
\hline Pinha & Annona squamosa & Annonaceae & Exótica & 2 & 3,0 & 85,00 & 27,06 & 12,30 \\
\hline
\end{tabular}


Seriguela

Sibipiruna

Tipuana

$\begin{array}{ll}\text { Spondias purpúrea } & \text { Anacardiáceas } \\ \text { Caesalpinia peltophoroides } & \text { Fabaceae } \\ \text { Tipuana tipu } & \text { Fabaceae }\end{array}$

Total $\begin{array}{llllll}\text { Exótica } & 1 & 1,5 & 140,00 & 44,60 & 12,80\end{array}$

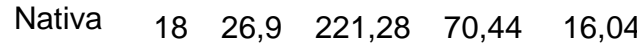

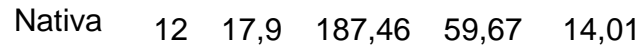

$67 \quad 100,0$

$\mathrm{N}=$ Números de indivíduos de cada espécie; \%=Porcentagem de indivíduos de cada espécie; DAP= Média do diâmetro a altura do peito dos indivíduos de cada espécie; $\mathrm{H}$ total= Média da altura total dos indivíduos de cada espécie; $\mathrm{CAP}=$ Média da circunferência a altura do peito. Fonte: Os Autores

Tabela 03: Dados das espécies arbóreas localizadas em cada área de uso comum da ETEC Prof. Dr. Antonio Eufrásio de Toledo.

\begin{tabular}{|c|c|c|c|c|c|c|c|c|}
\hline Nome popular & Nome científico & Família & Tipo & $\mathbf{N}$ & $\%$ & CAP & DAP & H total \\
\hline \multicolumn{9}{|c|}{ Prédios Administração e Salas de Aula 16.252,10 m² } \\
\hline Alfeneiro & Ligustrum lucidum & Oleaceae & Exótica & 15 & 13,6 & 0,83 & 0,27 & 11,70 \\
\hline Angico & Anadenanthera macrocarpa & Fabaceae & Nativa & 1 & 0,9 & 3,12 & 0,99 & 21,20 \\
\hline Areca-bambu & Dypsis lutescens & Arecaceae & Exótica & 2 & 1,8 & - & - & - \\
\hline Canivete & Erythrina velutina & Fabaceae & Nativa & 1 & 0,9 & 0,94 & 0,30 & 11,90 \\
\hline Cedro & Cedrela fissilis & Meliaceae & Nativa & 2 & 1,8 & 1,80 & 0,57 & 16,85 \\
\hline Cipreste & Cupressus sempervirens & Cupressaceae & Exótica & 1 & 0,9 & 0,48 & 0,15 & 13,70 \\
\hline Cyca & Cycas circinalis & Cycadaceae & Exótica & 1 & 0,9 & 0,95 & 0,30 & 6,70 \\
\hline Dracena & Dracaena fragrans & Ruscaceae & Exótica & 1 & 0,9 & 0,49 & 0,16 & 5,30 \\
\hline Espatódea & Spathodea campanulata & Bignoniaceae & Exótica & 4 & 3,6 & 0,85 & 0,27 & 14,68 \\
\hline Farinha-seca & Albizia hasslerii & Fabaceae & Nativa & 2 & 1,8 & 0,33 & 0,10 & 7,05 \\
\hline Ficus & Ficus benjamina & Moraceae & Exótica & 2 & 1,8 & 4,41 & 1,40 & 18,75 \\
\hline Flamboyant & Delonix regia & Fabaceae & Exótica & 2 & 1,8 & 1,39 & 0,44 & 9,15 \\
\hline Goiaba & Psidium guajava & Myrtaceae & Nativa & 1 & 0,9 & 0,54 & 0,17 & 6,70 \\
\hline Hibisco & Hibiscus rosa-sinensis & Malvaceae & Exótica & 1 & 0,9 & - & - & - \\
\hline Ipê Roxo & Handroanthus avellanedae & Bignoniaceae & Nativa & 3 & 2,7 & 1,19 & 0,38 & 15,58 \\
\hline Ipê-amarelo & Handroanthus alba & Bignoniaceae & Nativa & 9 & 8,2 & 0,41 & 0,13 & 9,71 \\
\hline Ipê-amarelo de Jardim & Tecoma stans & Bignoniaceae & Exótica & 2 & 1,8 & 0,34 & 0,11 & 6,85 \\
\hline Ipê-rosa & Tabebuia pentaphylla & Bignoniaceae & Nativa & 1 & 0,9 & 0,97 & 0,31 & 10,70 \\
\hline Ipê-roxo-bola & Tabebuia impetiginosa & Bignoniaceae & Exótica & 3 & 2,7 & 1,12 & 0,36 & 11,85 \\
\hline Jatobá & Hymenaea courbaril & Fabaceae & Nativa & 1 & 0,9 & 1,57 & 0,50 & 16,70 \\
\hline Jequitibá & Cariniana legalis & Lecythidaceae & Nativa & 1 & 0,9 & 0,81 & 0,26 & 13,70 \\
\hline Koelreuteria & Koelreuteria bipinnata & Sapindaceae & Exótica & 2 & 1,8 & 0,64 & 0,11 & 7,75 \\
\hline Mamão & Carica papaya & Caricaceae & Exótica & 1 & 0,9 & 0,72 & 0,23 & 6,70 \\
\hline Monguba & Pachira aquática & Malvaceae & Nativa & 4 & 3,6 & 0,90 & 0,29 & 7,95 \\
\hline Murta & Blepharocalyx salicifolius & Myrtaceae & Nativa & 2 & 1,8 & 0,57 & 0,18 & 6,65 \\
\hline Oiti & Licania tomentosa & Chrysobalanaceae & Nativa & 7 & 6,4 & 0,46 & 0,15 & 6,03 \\
\hline Paineira & Chorisia speciosa & Malvaceae & Nativa & 2 & 1,8 & 1,35 & 0,43 & 13,25 \\
\hline Palmeira/Tamareira & Phoenix dactylifera & Palmaceae & Exótica & 1 & 0,9 & 0,90 & 0,29 & 7,70 \\
\hline Palmeira-imperial & Roystonea oleracea & Arecaceae & Exótica & 2 & 1,8 & 1,29 & 0,41 & 12,45 \\
\hline Palmeira-pupunha & Bactris gasipaes & Arecaceae & Nativa & 1 & 0,9 & 0,43 & 0,14 & 8,70 \\
\hline Pata-de-vaca & Bauhinia forticata & Fabaceae & Nativa & 1 & 0,9 & 0,73 & 0,23 & 6,70 \\
\hline
\end{tabular}




\begin{tabular}{lllllllll} 
Pau-ferro & Caesalpinia ferrea & Fabaceae & Nativa & 1 & 0,9 & 2,30 & 0,73 & 19,70 \\
Pinus & Pinus taeda & Pinaceae & Exótica & 3 & 2,7 & 1,04 & 0,33 & 14,15 \\
Pitanga & Eugenia uniflora & Myrtaceae & Nativa & 1 & 0,9 & 0,30 & 0,10 & 4,50 \\
Saboneteiro & Sapindus saponaria & Sapindaceae & Nativa & 2 & 1,8 & 0,28 & 0,09 & 4,75 \\
Santa-barbara & Melia azedarach & Meliaceae & Exótica & 1 & 0,9 & 2,92 & 0,93 & 16,50 \\
Sete-copas & Lecythis pisonis & Combretaceae & Exótica & 3 & 2,7 & 2,06 & 0,66 & 22,32 \\
Sibipiruna & Caesalpinia peltophoroides & Fabaceae & Nativa & 7 & 6,4 & 2,21 & 0,70 & 17,62 \\
Sobrasil & Colubrina glandulosa & Rhamnaceae & Nativa & 1 & 0,9 & 0,73 & 0,23 & 14,90 \\
Tamarindo & Tamarindus indica & Fabaceae & Exótica & 1 & 0,9 & 1,58 & 0,50 & 13,30 \\
Tipuana & Tipuana tipu & Fabaceae & Nativa & 11 & 10,0 & 1,69 & 0,54 & 17,11 \\
& & & & 110 & 100 & - & - & - \\
\hline
\end{tabular}

$\mathrm{N}=$ Números de indivíduos de cada espécie; \%=Porcentagem de indivíduos de cada espécie; DAP= Média do diâmetro a altura do peito dos indivíduos de cada espécie; $\mathrm{H}$ total= Média da altura total dos indivíduos de cada espécie; CAP= Média da circunferência a altura do peito. Fonte: Os Autores

Tabela 04: Dados das espécies arbóreas localizadas em cada área de uso comum da ETEC Prof. Dr. Antonio Eufrásio de Toledo.

\begin{tabular}{|c|c|c|c|c|c|c|c|c|}
\hline Nome popular & Nome científico & Família & Tipo & $\mathbf{N}$ & $\%$ & CAP & DAP & $\mathbf{H}$ total \\
\hline \multicolumn{9}{|c|}{ Área Horta $5000 \mathrm{~m}^{2}$} \\
\hline Abacate & Persea americana & Lauraceae & Exótica & 1 & 1,4 & 123,33 & 39,26 & 9,30 \\
\hline Abiurana & Chrysophyllum sp & Sapotaceae & Exótica & 1 & 1,4 & 35,50 & 11,30 & 5,33 \\
\hline Acerola & Malphighia glabra & Malpighiaceae & Exótica & 7 & 9,9 & - & - & - \\
\hline Ameixa japonesa & Prunus salicina & Rosáceas & Exótica & 1 & 1,4 & 56,00 & 17,83 & 7,62 \\
\hline Amora & Morus nigra & Moraceae & Exótica & 14 & 19,7 & 64,65 & 20,58 & 6,04 \\
\hline Cajamanga & Spondias dulcis & Anacardiaceae & Exótica & 2 & 2,8 & 88,50 & 28,17 & 7,50 \\
\hline Calabura & Muntingia calabura & Muntingiaceae & Exótica & 1 & 1,4 & 28,50 & 9,07 & 5,50 \\
\hline Coqueiro & Cocos nucifera & Arecaceae & Exótica & 1 & 1,4 & 6,70 & 2,13 & 5,00 \\
\hline Flamboyant & Delonix regia & Fabaceae & Exótica & 1 & 1,4 & 176,00 & 56,02 & 10,00 \\
\hline Goiaba vermelha & Psidium sp & Myrtaceae & Nativa & 1 & 1,4 & 49,00 & 15,60 & 6,00 \\
\hline Jabuticaba & Myrciaria cauliflora & Myrtaceae & Nativa & 1 & 1,4 & 118,00 & 37,56 & 4,95 \\
\hline Jambo & Eugenia jambolana & Myrtaceae & Exótica & 4 & 5,6 & 147,67 & 47,00 & 6,23 \\
\hline Jambolão & Syzygium cumini & Myrtaceae & Exótica & 2 & 2,8 & 88,56 & 28,19 & 7,43 \\
\hline Jambolão branco & Syzygium aqueum & Myrtaceae & Exótica & 6 & 8,5 & 66,70 & 21,23 & 7,60 \\
\hline Jambolão roxo & Syzygium jambolanum & Myrtaceae & Exótica & 1 & 1,4 & 137,35 & 43,72 & 8,00 \\
\hline Mamão & Carica papaya & Caricaceae & Exótica & 7 & 9,9 & 31,14 & 9,91 & 2,99 \\
\hline Murta-de-cheiro & Murraya paniculata & Rutaceae & Exótica & 14 & 19,7 & 46,08 & 14,67 & 5,98 \\
\hline Pitanga & Eugenia uniflora & Myrtaceae & Nativa & 1 & 1,4 & 119,00 & 37,88 & 2,64 \\
\hline Santa bárbara & Melia azedarach & Meliaceae & Exótica & 1 & 1,4 & 59,50 & 18,94 & 5,75 \\
\hline Uva japonesa & Hovenia dulcis & Rhamnaceae & Exótica & 1 & 1,4 & 65,00 & 20,69 & 9,00 \\
\hline Total & & & & 71 & 100 & - & - & - \\
\hline
\end{tabular}




\begin{tabular}{lllllllll}
\hline \multicolumn{1}{c}{ Área Viveiro Florestal $\mathbf{6 0 0} \mathbf{~ m}^{\mathbf{2}}$} & & & & \\
\hline Abacate & Persea americana & Lauraceae & Exótica & 1 & 2,27 & 97,00 & 30,88 & 9,36 \\
Calabura & Muntingia calabura & Muntingiaceae & Nativa & 12 & 27,27 & 71,20 & 22,66 & 18,38 \\
Eucalipto & Eucalyptus sp. & Myrtaceae & Exótica & 27 & 61,36 & 66,78 & 21,26 & 35,97 \\
Mangueira & Mangifera indica & Anacardiaceae & Exótica & 3 & 6,82 & 147,00 & 46,79 & 30,90 \\
Sete copas & Lecythis pisonis & Combretaceae & Exótica & 1 & 2,27 & 103,00 & 32,79 & 15,30 \\
Total & & & & 44 & 100 & - & - & - \\
\hline
\end{tabular}

$\mathrm{N}=$ Números de indivíduos de cada espécie; \%=Porcentagem de indivíduos de cada espécie; DAP= Média do diâmetro a altura do peito dos indivíduos de cada espécie; $\mathrm{H}$ total= Média da altura total dos indivíduos de cada espécie; CAP= Média da circunferência a altura do peito. Fonte: Os Autores

\section{Espécies de arvores Nativas e Exóticas}

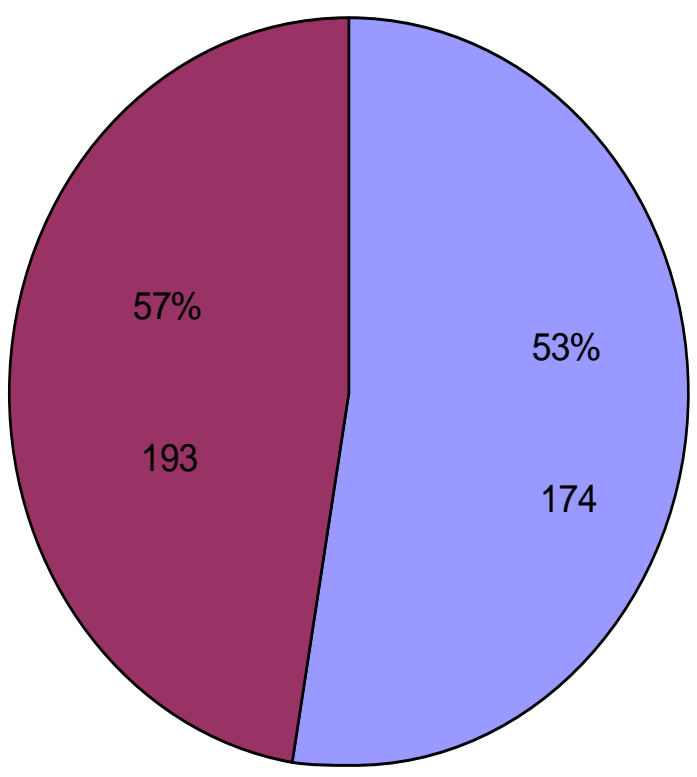

$\square$ 1ํㅡㄹótica

$\square 2^{\circ}$ Nativa

Figura 4: Espécies de arvores Nativas e Exóticas (\%) na ETEC de Presidente Prudente. Fonte: Os autores

No inventário de plantas arbóreas foram registrados 367 indivíduos, sendo 193 de espécies exóticas (57\%) e 174 de nativas (53\%) (Figura 4). As famílias Fabaceae $(23,4 \%)$, Myrtaceae $(19.9 \%)$ e Bignoniaceae $(6,8 \%)$ foram as mais freqüentes dentre as plantas (Figura 5). 


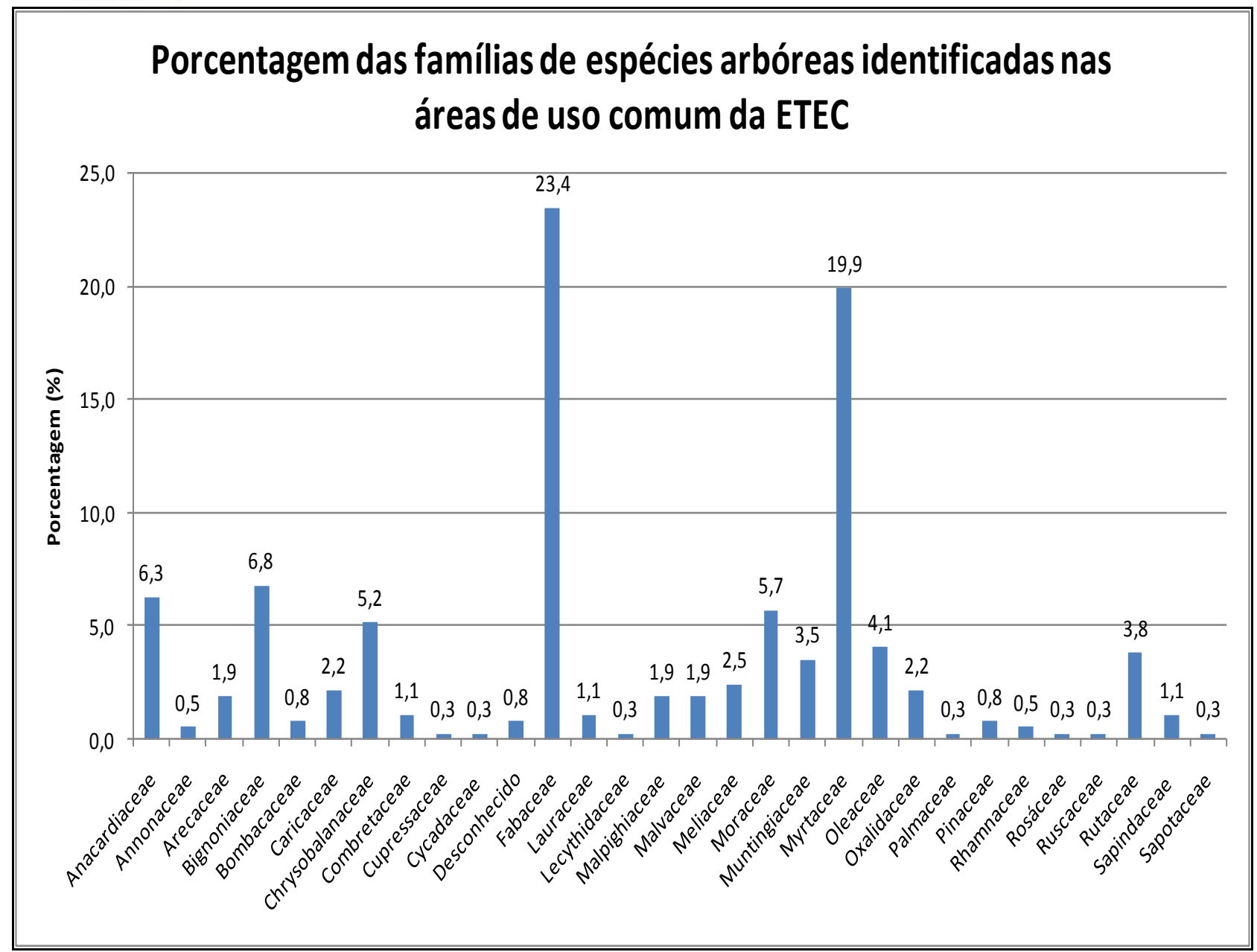

Figura 5: Porcentagem das famílias de espécies arbóreas localizadas nas áreas de uso comum da ETEC de Presidente Prudente. Fonte: Os autores.

A diversidade de aves encontradas se deve ao fato da escola possuir diferentes habitats tais como áreas de Sistema Agro Florestal (SAF), campos abertos, bordadura de mata, áreas de preservação permanente, horta, entre outras, além de fazer divisa com o Parque Ecológico Cidade da Criança. Outro fator que contribui para atrair a avifauna é grande diversidade de árvores frutíferas.

Entre os ambientes analisados, o que revelou maior quantidade de espécies distintas foi o campo aberto. Tal riqueza pode ser explicada pelo comportamento alimentar das próprias aves, como o vira-bosta (Molothrus bonariensis) e o quero-quero (Vanellus chilensis) que ficam em áreas de campo aberto alimentando-se de insetos, e os Falconiformes encontrados no local. 
No local onde se desenvolveu o presente trabalho foram encontradas aves de médio porte como o anu-preto (Crotophaga ani) e o anu-branco (Guira guira), o que foi verificado também por Straube et al. (2005) em regiões periantrópicas de sua área estudo, realizado no Parque Estadual do Cerrado no Estado do Paraná.

Uma das aves freqüentemente avistadas nas áreas de campo aberto foi a garça-vaqueira (Bubulcus ibis), membro da família Ardeidae, conhecida por acompanhar rebanhos bovinos já que costuma se alimentar dos insetos que parasitam o gado, com quem estabelecem uma relação de benefício mútuo (BELLA et al, 2004; MENEZES et al, 2004).

Silva e Nakano (2008) obtiveram resultados semelhantes, verificando a presença de espécies como a garça vaqueira, garça branca grande, anu branco e anu preto entre outras, em seu trabalho desenvolvido em uma área de cerrado no Bairro do Central Parque, Município de Sorocaba, São Paulo, Brasil.

No trabalho de Matos (2011), realizado no campus da Faculdade de Ciências e Tecnologia de Presidente Prudente (FCT/UNESP) também são descritos resultados semelhantes, o que indica características comuns entre as duas áreas de estudo.

Sendo assim, foi possível estabelecer um check-list das aves da ETEC Prof. Dr. Antonio Eufrásio de Toledo, o que permitirá um acompanhamento da diversidade ao longo do tempo. A partir desse levantamento preliminar da avifauna e da flora arbórea espera-se propor a introdução de novas espécies arbóreas, favorecendo um enriquecimento do ambiente. Desta forma permitindo uma maior ocorrência de aves na ETEC Prof. Dr. Antonio Eufrásio de Toledo.

\section{CONCLUSÃO}

Tais dados preliminares revelam a importância da conservação da área verde para a manutenção da avifauna que adotou a ETEC como refúgio, por possuir recursos como alimento, abrigo e locais para reprodução em meio à urbanização e crescimento horizontal da cidade de Presidente Prudente. 


\section{REFERÊNCIAS}

ANDRADE, M. A. de. A Vida das Aves: Introdução à Biologia e Conservação. Belo Horizonte, Minas Gerais: Littera Maciel Ltda. 1993.

BAPTISTA, L. F.; TRAIL, P. W.; HORBLIT, H. M. Family Columbidae. In: DEL HOYO, J.; ELLIOTT, A. \& SARGATAL, J. Handbook of the Birds of the World. v. 4. Sandgrouse to Cuckoos. Lynx Edicions, Barcelona, España. 1997. p. 60-243.

BELLA, S. D.; AZEVEDO, S. M. Jr. Considerações sobre a ocorrência da garça vaqueira, Bubulcus íbis (Linnaeus) (Aves, Ardeidae), em Pernambuco, Brasil. Rev. Bras. De Zoologia, Curitiba. v. 1, n. 21, p. 57-63, 2004.

CLEMENTS; J. F. The Clements Checklist of Birds of the World. 6 ed. Cornell University Press, 2007.

CBRO - Comitê Brasileiro de Registros Ornitológicos - Listas de Aves do Brasil. Versão 25/01/2011. Disponível em http://www.cbro.org.br/CBRO/listabr.htm Acesso em: 05 agos. 2013.

D'ANGELO-NETO, S.; VENTURINI, N.; OLIVEIRA FILHO, A.T.; COSTA, F.A. F. Avifauna de quatro fisionomias florestais de pequeno tamanho no Campus da UFLA. Revista Brasileira de Biologia, v.58, p.463-472, 1998.

FONTANA, C. S.; BENCKE, G. A.; REIS, E R. E. (ORG.). Livro Vermelho da Fauna Ameaçada de Extinção no Rio Grande do Sul. Porto Alegre: EDIPUCRS. 2003. 632p.

GIMENES, M. R. \& ANJOS. L. Distribuição espacial de aves em um fragmento florestado campus da Universidade Estadual de Londrina, Norte do Paraná, Brasil Revista Brasileira de Zoologia. 17 (1): 263 - 271, 2000.

GRILL, F. B. Ornithology. 2 ed. New York: W.H. Freeman and Company, 1995.

HICKMAN, Jr. C. P.; ROBERTS, L. S.; LARSON, A. Princípios Integrados de Zoologia. 11 ed. Rio de Janeiro: Guanabara Koogan, 2004. p.571.

MARÇAL JÚNIOR, O.; FRANCHIN, A. G. Aves, do latim avis. In: Del Claro, K.; Prezoto, F. (org.). As distintas Faces do Comportamento Animal. Jundiaí: Livraria Conceito. 2003. p. 105-119. 
MATOS, R. J. Estudo biogeográfico: Levantamento da Avifauna existente no campus da faculdade de ciências e tecnologia de Presidente Prudente (FCT/UNESP). Revista Formação Online, n. 18, volume 2, p. 66-78, jul./dez., 2011.

MENEZES, I. R.; MEDEIROS, F. P. M.; ALBUQUERQUE, H. N.; ALBUQUERQUE, I.C. S.; BARBOSA, A. R.; BARBOZA, R. R. D. Comportamento Alimentar da Garça Vaqueira, Bubulcus íbis (LINNAEUS 1758) (AVES: ARDEIDAE): Um Estudo Preliminar. Rev. de Biologia e Ciências da Terra. v. 4, n. 1, 2004.

PROBIO. Projeto de conservação e utilização sustentável da diversidade biológica brasileira: relatório de atividades. Brasília: Ministério do Meio Ambiente, 2002. Biodiversidade - Brasil. I. Ministério do Meio Ambiente. 2002.

RIDGELY, R. S.; TUDOR, G. The Birds of South America. 2 ed. Vol.1. Oxford University Press, 1994.

RIECHELMANN, C. C. Rurbanização, desenvolvimento e vida: o caso do assentamento Nova Esperança I, do MST, em macrozona de expansão urbana de São José dos Campos - Perspectivas para o Planejamento Urbano e Regional. Univap, p. 433, 2006.

SICK, H. Ornitologia Brasileira. Rio de Janeiro: Nova Fronteira, 1997. p. 912.

SILVA, J. M. C.; BATES, J. M. Biogeographic patterns and conservation in the South American Cerrado: a tropical savanna Hotspot. BioScience, Albertson, v. 52, n. 3, p. 225-233, 2002.

SILVA, L. A. C.; NAKANO, C. A. Avifauna em uma Área de Cerrado no Bairro do Central Parque, Município de Sorocaba, São Paulo, Brasil. Revista Eletrônica de Biologia. Volume 1 (1): 36-61, 2008.

SILVA, W. R.; ALEIXO, A. L. P. Estudo da diversidade de espécies de aves do Estado de São Paulo: versão preliminar. Depto. Zoologia - Instituto de Biologia -UNICAMP, SP, 1996.

StRAUBE, F. C.; URBEN, A. F.; GATTO, C. A Avifauna do Parque Estadual do Cerrado (Jaguariaíva, Paraná) e a Conservação do Cerrado em seu Limite Meridional de Ocorrência. Revista Atualidades Ornitológicas. n. 127, p. 29. 2005.

VOGEL, H. F.; METRI, R.; ZAWADZKI, C. H.; MOURA. M. O. Avifauna from a campus of Universidade Estadual do Centro-Oeste, Guarapuava, Paraná State, Brazil Acta Scientiarum. Biological Sciences. Maringá, v. 33, n. 2, p. 197-207, 2011. 
VUILLEUMIER, F. Birds. In: BURNIE, C. et al. Illustrated Encyclopedia of Animals. London: Dorling Kindersley Limited, 2008.

WILLIS, E.O. Effects of a cold wave on an Amazonian avifauna in the upper Paraguay drainage, Western Mato Grosso, and suggestions on Oscine-Suboscine relationships. Acta Amazonica, v.6, p.379-394, 1976. 